\title{
An Adaptive Control Method for Magnetostrictive Transducers with Hysteresis
}

\author{
James M. Nealis and Ralph C. Smith \\ Center for Research in Scientific Computation \\ North Carolina State University \\ Raleigh, NC 27695 \\ jmnealis@eos.ncsu.edu, rsmith@eos.ncsu.edu
}

\begin{abstract}
This paper addresses the development of an adaptive control strategy for regulating the output of a prototypical magnetostrictive transducer. The application is a generalization of an industrial process in which the magnetostrictive transducer is used to drive a cutting head in a high speed, high accuracy mode of operation. To attain the required stroke and force requirements, the transducer must operate in a regime in which the relation between input currents and output strains or forces exhibits significant hysteresis and constitutive nonlinearities including saturation effects. A variety of techniques for modeling the hysteresis and transducer dynamics are discussed and a model reference adaptive control (MRAC) strategy based on a Preisach model is developed. The performance of the control method is illustrated through a numerical example.
\end{abstract}

\section{Introduction}

Magnetostrictive transducers provide unique actuator capabilities in a number of automotive and industrial applications due to their broad bandwidth and capability for generating large forces. A prototypical process in which magnetostrictive transducers have been proven advantageous is the milling of out-of-round products at high speeds subject to micron-level tolerances. As detailed in [8], a magnetostrictive milling device has recently been developed by Etrema Products ${ }^{1}$ with the goal of achieving \pm 1 micron cutting tolerances while operating at $3000 \mathrm{rpm}$. It is this application that we consider as a prototype for adaptive control design.

To achieve bi-directional strokes, it is necessary to bias magnetostrictive transducers through either an applied DC current or a surrounding magnet. Even in this biased state, however, the transducers exhibit hysteresis and constitutive nonlinearities which must be accommodated in both models and control designs to meet

\footnotetext{
${ }^{1}$ Etrema Products, Inc., 2500 North Loop Drive, Ames, IA 50010
}

the speed and accuracy specifications. This paper addresses the development of an adaptive control method to achieve this goal.

To place the model and control design in context, we first summarize some existing techniques for characterizing magnetostrictive transducers operating in nonlinear and hysteretic regimes. Two modeling techniques can be roughly categorized as Preisach characterizations $[1,6,7]$ and quasi-macroscopic models based on domain properties of the materials $[2,3,11]$. The former approach represents hysteresis through expansions based on a variety of multivalued kernels which, due to their global nature, directly enforce minor loop closure and loop congruency. The disadvantage of the technique derives from the lack of an energy basis for the method; hence it can be difficult to incorporate general frequency-dependence and the representation of asymmetric loops can produce high-order models with nonphysical parameters. In contrast, the domain wall approach employs magnetoelastic energy relations to derive models in which the magnetization generated by an input field is evolved through the solution of a loworder ordinary differential equation. Due to its energy foundation, the domain model directly incorporates frequency-dependence and extensions to incorporate thermal-dependence are under development.

In this paper, we consider the development of a model reference adaptive control (MRAC) for magnetostrictive transducers operating at fixed, high frequency conditions. We employ the Preisach-based techniques from $[9,10]$ since, for the conditions under consideration, the Preisach model provides sufficient accuracy and admits a linear parameterization. The primary extension that we provide to the theory of $[9,10]$ is the incorporation of the full transducer model to characterize both the magnetic and elastic properties of the transducer.

The transducer model, hysteresis model and model inverse are summarized along with commensurate numerical techniques in Section 2. The MRAC design is outlined in Section 3 and illustrated through a numerical example in Section 4. 


\section{Transducer Model and Inverse}

For the purpose of model development, we consider the transducer depicted in Figure 1. This design was employed in the motivating milling device and is representative of designs employed in a number of other industrial and academic applications. Forces and strains are generated through the rotation of dipoles in the Terfenol-D core in response to current-induced fields in the surrounding solenoid. Biases required to achieve bidirectional strains and forces are provided by the permanent magnet. The compression bolt and prestress spring keep the rod in compression and further align dipoles to increase outputs due to dipole rotation.

The model will be constructed in two steps. In the first, the dynamics of the Terfenol-D rod will be quantified through the development of a PDE model with general inputs provided by magnetic field induced strains. The nonlinear and hysteretic relation between input currents and the generated magnetization will then be quantified through a Preisach characterization to provide a complete model for the transducer dynamics.

\subsection{Transducer Model}

We consider the Terfenol-D rod to have length $L$, cross-sectional area $A$, density $\rho$, Young's modulus $E$ and damping coefficient $c_{D}$. The rod is assumed to be fixed at the left end $(x=0)$ and the right end $(x=L)$ has an attached end mass $M_{L}$ and is constrained by the prestress spring which is assumed to have stiffness $k_{L}$ and damping coefficient $c_{L}$.

Under the assumption of linear elasticity and KelvinVoigt damping, the stress $\sigma$ at any point in the rod is given by

$$
\sigma(t, x)=E \frac{\partial w}{\partial x}(t, x)+c_{D} \frac{\partial^{2} w}{\partial x \partial t}(t, x)-E \lambda(t)
$$

where $w(t, x)$ denotes the longitudinal displacement and $\lambda$ is the magnetostriction, or free strains, due to dipole rotation. As illustrated in [3], the magnetostriction has the general formulation $\lambda(t, x)=\frac{3 \lambda_{s}}{2 M^{2}} M^{2}(t, x)$ where $\lambda_{s}$ and $M_{s}$ respectively denote the saturation magnetostriction and magnetization and $M(t, x)$ reflects the spatial variability in the magnetization due to end effects in the rod. Through judicious transducer design, however, end effects can be minimized which motivates the assumption of spatially invariant magnetostriction in (1). Furthermore, to achieve bi-direction strains and forces, it is necessary to bias the magnetization about a level $M_{0}$ generated by the permanent magnet. For low strain, large force applications, reasonable accuracy can be obtained with the linearization

$$
\lambda(t)=\frac{3 \lambda_{s}}{2 M_{s}^{2}}\left[2 M(t) M_{0}-M_{0}^{2}\right]
$$

Wound Wire Solenoid

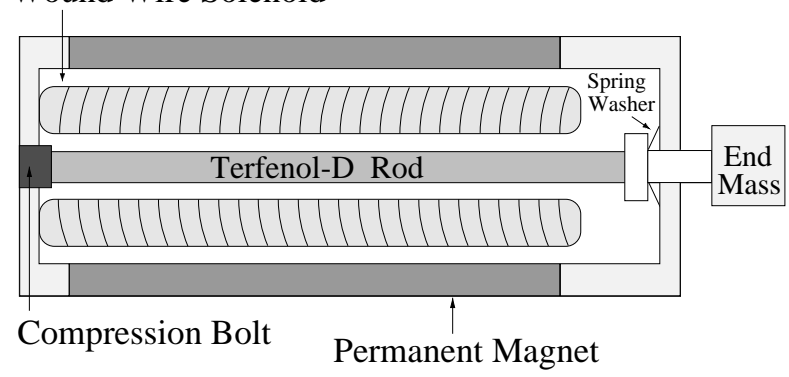

Figure 1. Cross section of the Terfenol-D magnetostrictive transducer.

and it is this regime that we consider. For large strains, one must retain the biased quadratic model developed in [3].

To obtain a modeling PDE, force balancing is employed to obtain

$$
\rho A \frac{\partial^{2} w}{\partial t^{2}}=\frac{\partial N_{t o t}}{\partial x}
$$

where the resultant is specified by

$$
N_{t o t}=E A \frac{\partial w}{\partial x}+c_{D} A \frac{\partial^{2} w}{\partial x \partial t}-E A \lambda .
$$

To obtain appropriate boundary conditions, we first note that $w(t, 0)=0$. Force balancing at $x=L$ yields

$$
N_{t o t}(L, t)=-k_{L} w(t, L)-c_{L} \frac{\partial w}{\partial t}(t, L)-M_{L} \frac{\partial^{2} w}{\partial t^{2}}(t, L) .
$$

To pose the PDE (3) in a form which facilitates approximation, we consider a weak form of the model with state space $X=L^{2}(0, L)$ and the space of test functions $V=H_{L}^{1}(0, L) \equiv\left\{\phi \in H^{1}(0, L) \mid \phi(0)=0\right\}$. Multiplication by test functions followed by integration then yields the weak form

$$
\begin{aligned}
\int_{0}^{L} \rho A & \frac{\partial^{2} w}{\partial t^{2}} \phi d x=-\int_{0}^{L} E A \frac{\partial w}{\partial x} \frac{\partial \phi}{\partial x} d x \\
& -\int_{0}^{L} c_{D} A \frac{\partial^{2} w}{\partial x \partial t} \frac{\partial \phi}{\partial x} d x+\int_{0}^{L} E A \lambda \frac{\partial \phi}{\partial x} d x \\
& -\left(k_{L} w+c_{L} \frac{\partial w}{\partial t}+M_{L} \frac{\partial^{2} w}{\partial t^{2}}\right)(t, L) \phi(L)
\end{aligned}
$$

which must be satisfied for all $\phi \in V$.

For either simulation purposes or control implementation, it is necessary to discretize the infinite dimensional model (4). To define a finite element discretization in space, we consider a uniform partition of the interval $[0, L]$ and consider a basis $\left\{\phi_{i}\right\}_{i=1}^{N}$ comprised of linear splines (see [5] for details).

The solution $w(t, x)$ to $(4)$ is then approximated by

$$
w^{N}(t, x)=\sum_{j=1}^{N} w_{j}(t) \phi_{j}(x) .
$$


The projection of the problem (4) into the finite dimensional subspace $H^{N}=\operatorname{span}\left\{\phi_{i}\right\}_{i=1}^{N} \subset H_{L}^{1}(0, L)$ yields the second-order semidiscrete system

$$
Q \ddot{\vec{w}}(t)+C \dot{\vec{w}}(t)+K \vec{w}(t)=\vec{b}(t)
$$

where $\vec{w}(t)=\left[w_{1}(t), \cdots, w_{N}(t)\right]$. Descriptions of the mass, damping and stiffness matrices $Q, C$ and $K$ can be found in [3, 4]. The input vector has the form $\vec{b}(t)=\vec{\beta} u(t)$ where $\vec{\beta}_{i}=E A \frac{3 \lambda_{s} M_{0}}{M_{s}^{2}} \int_{0}^{L} \frac{d \phi_{i}}{d x} d x$ and

$$
u(t)=M(v(t))
$$

denotes the hysteretic relation between the control input $v(t)$ to the transducer and the resulting magnetization as depicted in Figure 2. For experimental implementation, the input $v(t)$ consists of the current $\mathcal{I}(t)$ applied to the solenoid which generates a field $H(t)=\mathcal{N} \mathcal{I}(t)$, where $\mathcal{N}$ denotes the number of coils per unit length in the solenoid.

To formulate the system in first-order form, we let $\vec{x}(t)=[\vec{w}(t), \dot{\vec{w}}(t)]^{T}$ and define

$$
A=\left[\begin{array}{cc}
0 & I \\
-Q^{-1} K & -Q^{-1} C
\end{array}\right], B=\left[\begin{array}{c}
0 \\
Q^{-1} \vec{\beta}
\end{array}\right]
$$

to obtain

$$
\begin{aligned}
\dot{\vec{x}}(t) & =A \vec{x}(t)+B u(t) \\
\vec{x}(0) & =\vec{x}_{0} .
\end{aligned}
$$

A typical means of observation is to measure the displacements at the rod tip. This yields the $1 \times 2 N$ observation vector

$$
C=[0, \cdots, 0,1,0, \cdots, 0]
$$

where the 1 occurs in the $N^{t h}$ position.

The transfer function for the system (7) is given by

$$
G(s)=C(s I-A)^{-1} B=k_{p} \frac{Z(s)}{P(s)} .
$$

This defines the system in a manner appropriate for subsequent adaptive control design.

\subsection{Hysteresis Model}

To characterize the hysteretic relation (6) between the applied current $v(t)=\mathcal{I}(t)$ and the resulting magnetization $M$, we employ a piecewise linear Preisach model. The following discussion summarizes the hysteresis model from $[9,10]$ and the reader is directed to those sources for additional details.

In this model, a hysteresis loop is defined by the two half-lines

$$
u(t)=\left\{\begin{array}{l}
m_{t} v(t)+c_{t}, v(t)>v_{1}=\frac{c_{t}+m_{\ell} c_{\ell}}{m_{\ell}-m_{t}} \\
m_{b} v(t)+c_{b}, v(t)<v_{2}=\frac{c_{b}+m_{r} c_{r}}{m_{r}-m_{b}}
\end{array}\right.
$$

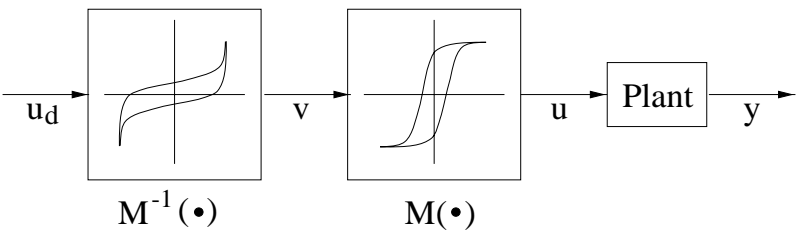

Figure 2. Plant with actuator hysteresis $M(\cdot)$ and inverse compensator $M^{-1}(\cdot)$ for the hysteresis.

and two line segments

$$
u(t)=\left\{\begin{array}{l}
m_{r}\left(v(t)-c_{r}\right), v_{2} \leq v(t)<v_{3}=\frac{c_{t}+m_{r} c_{r}}{m_{r}-m_{t}} \\
m_{\ell}\left(v(t)-c_{\ell}\right), \frac{c_{b}+m_{\ell} c_{\ell}}{m_{\ell}-m_{b}}=v_{4}<v(t) \leq v_{1}
\end{array}\right.
$$

where $m_{i}$ denotes slopes and $c_{i}$ denotes crossing parameters. The subscripts $i=\ell, r, b$ and $t$ respectively indicate left, right, bottom and top. For the given application, symmetry dictates that $m_{t}=m_{b}, c_{t}=-c_{b}>0$, $m_{r}=m_{\ell}$ and $c_{r}=-c_{\ell}>0$; details regarding the asymmetric case can be found in [10]. Finally, $v_{1}, v_{2}, v_{3}$ and $v_{4}$ indicate the values of $v(t)$ at the corners of the hysteresis loop as depicted in Figure 3.

The time derivatives of $u(t)$ and $v(t)$ are constant along the line segments with $\dot{v}>0$ and $\dot{u}>0$ for the segment $u(t)=m_{r}\left(v(t)-c_{r}\right)$. On the half line $u(t)=m_{t} v(t)+c_{t}, \dot{v}<0$ and $\dot{u}<0$ if $v(t)<v_{3}$ but $\dot{v}$ and $\dot{u}$ need only be the same sign if $v(t)>v_{3}$. Similar conditions apply for the remaining segments of the hysteresis model.

The line segments for inner hysteresis loops are defined by

$$
u(t)=\left\{\begin{array}{l}
m_{t} v(t)+c_{d}(t), \dot{v}<0 \\
m_{b} v(t)+c_{u}(t), \dot{v}>0
\end{array}\right.
$$

where $c_{d}$ and $c_{u}$ are the intercepts for the downward and upward inner loop segments, respectively. As detailed

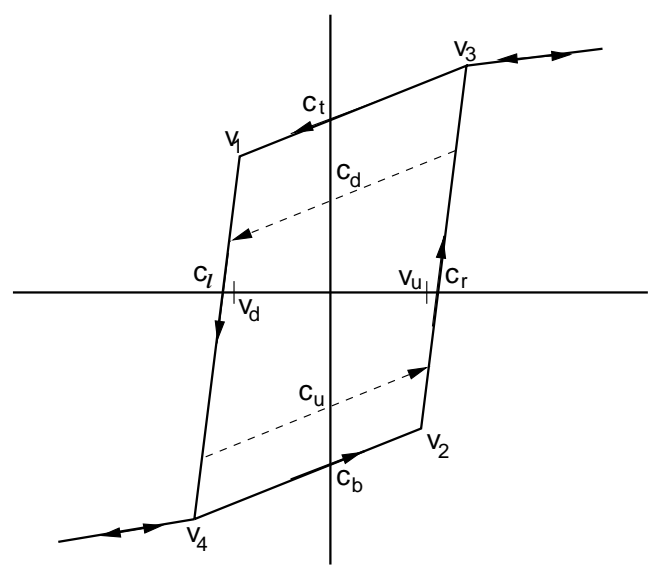

Figure 3. Piecewise linear Preisach hysteresis model. 
in [10], the combination of half-line and segment relations yields a dynamic model quantifying $\dot{u}(t)$ in terms of $\dot{v}(t)$ as a function of location within the hysteresis landscape.

To obtain a discrete-time model suitable for numerical or experimental implementation, time derivatives are replaced by comparing $v(t)$ with $v_{d}$ and $v_{u}$ where $v_{4} \leq v_{d} \leq v_{u} \leq v_{3}$ and

$$
v_{d}=\frac{m_{\ell} c_{\ell}+c_{d}}{m_{\ell}-m_{t}}, \quad v_{u}=\frac{m_{r} c_{r}+c_{u}}{m_{r}-m_{b}}
$$

(see Figure 3). Due to symmetry, $m_{t}=m_{b}$ and the constants $c_{d}$ and $c_{u}$ are given by

$$
c_{d}=c_{u}=u\left(t_{k-1}\right)-m_{t} v\left(t_{k-1}\right) .
$$

The discrete-time hysteresis model is then given by

$$
u\left(t_{k}\right)= \begin{cases}u\left(t_{k-1}\right) & \text { if } v\left(t_{k}\right)=v\left(t_{k-1}\right) \\ m_{t} v\left(t_{k}\right)+c_{t} & \text { if } v\left(t_{k}\right) \leq v_{3}, \text { or } \\ & \text { if } m_{t}<m_{b}, \\ & u\left(t_{k-1}\right)=m_{t} v\left(t_{k-1}\right)+c_{t} \\ & \text { and } v\left(t_{k-1}\right)<v\left(t_{k}\right)<v_{3} \\ m_{b} v\left(t_{k}\right)+c_{b} & \text { if } v\left(t_{k}\right) \leq v_{4}, \text { or } \\ & \text { if } m_{t}>m_{b}, \\ & u\left(t_{k-1}\right)=m_{b} v\left(t_{k-1}\right)+c_{b} \\ & \text { and } v_{4}<v\left(t_{k}\right)<v\left(t_{k-1}\right) \\ m_{t} v\left(t_{k}\right)+c_{d} & \text { if } v_{d}<v\left(t_{k}\right)<v\left(t_{k-1}\right) \\ m_{b} v\left(t_{k}\right)+c_{u} & \text { if } v_{d}\left(t_{k-1}\right)<v\left(t_{k}\right)<v_{u} \\ m_{\ell}\left(v\left(t_{k}\right)-c_{\ell}\right) & \text { if } v_{d} \geq v\left(t_{k}\right) \geq v_{4} \\ m_{r}\left(v\left(t_{k}\right)-c_{r}\right) & \text { if } v_{u} \leq v\left(t_{k}\right) \leq v_{3} .\end{cases}
$$

\subsection{Inverse Hysteresis Model}

The Preisach model outlined in the previous section provides a characterization of the hysteresis inherent to the magnetostrictive transducer. In this section, we summarize the construction of an inverse which can be employed as a filter to compensate, or approximately compensate, for the hysteresis inherent to the transducer. Because of the piecewise linear nature of the hysteresis model, and hence its inverse, the construction of the inverse is analogous to that of the model and we summarize only the main features pertinent to control design. Details can be found in $[9,10]$.

Because one goal in adaptive control design is to estimate parameters in the inverse, we let $\widehat{m}_{t}(t)$ denote the time-varying estimate of $m_{t}$ with similar notation for the estimates of the remaining parameters. As depicted in Figure 2, the inverse model takes as input the desired control $u_{d}$ and outputs $v$ which is subsequently applied as input to the hysteretic transducer.
As detailed in $[9,10]$, the inverse model with fixed parameter estimates can be defined by the two half-lines

$$
v(t)=\left\{\begin{array}{lll}
\frac{1}{\widehat{m}_{t}}\left(u_{d}(t)-\widehat{c}_{t}\right) & , \quad u_{d}(t)>u_{1} \\
\frac{1}{\widehat{m}_{b}}\left(u_{d}(t)-\widehat{c}_{b}\right) & , \quad u_{d}(t)<u_{2}
\end{array}\right.
$$

and two line segments

$$
v(t)=\left\{\begin{array}{lll}
\frac{1}{\widehat{m}_{r}} u_{d}(t)+\widehat{c}_{r} & , \quad u_{2} \leq u_{d}(t)<u_{3} \\
\frac{1}{\widehat{m}_{\ell}} u_{d}(t)+\widehat{c}_{\ell} & , \quad u_{4}<u_{d}(t) \leq u_{1}
\end{array}\right.
$$

where the corners of the enclosed region are

$$
\begin{array}{rlrl}
u_{1}=\frac{\widehat{m}_{\ell}\left(\widehat{m}_{t} \widehat{c}_{t}+\widehat{c}_{t}\right)}{\widehat{m}_{\ell}-\widehat{m}_{t}} & , & u_{2}=\frac{\widehat{m}_{r}\left(\widehat{m}_{b} \widehat{c}_{r}+\widehat{c}_{b}\right)}{\widehat{m}_{r}-\widehat{m}_{b}} \\
u_{3}=\frac{\widehat{m}_{r}\left(\widehat{m}_{t} \widehat{c}_{r}+\widehat{c}_{t}\right)}{\widehat{m}_{r}-\widehat{m}_{t}} \quad, \quad u_{4}=\frac{\widehat{m}_{\ell}\left(\widehat{m}_{b} \widehat{c}_{\ell}+\widehat{c}_{b}\right)}{\widehat{m}_{\ell}-\widehat{m}_{b}} .
\end{array}
$$

The conditions on the temporal derivatives of $u_{d}$ and $v$ are analogous to those for the hysteresis model.

To characterize the discrete-time inverse, we define the $u_{d}$-intercepts

$$
\begin{aligned}
& \widehat{c}_{d}\left(t_{k}\right)=u_{d}\left(t_{k-1}\right)-\widehat{m}_{t} v\left(t_{k-1}\right) \\
& \widehat{c}_{u}\left(t_{k}\right)=u_{d}\left(t_{k-1}\right)-\widehat{m}_{b} v\left(t_{k-1}\right)
\end{aligned}
$$

along with the points

$$
\begin{aligned}
& u_{d r}\left(t_{k}\right)= \begin{cases}\frac{\widehat{m}_{r}\left(\widehat{m}_{t} \widehat{c}_{r}+\widehat{c}_{d}\right)}{\widehat{m}_{r}-\widehat{m}_{t}} & , u_{d}\left(t_{k-1}\right)>u_{d}\left(t_{k}\right) \\
\frac{\widehat{m}_{r}\left(\widehat{m}_{b} \widehat{c}_{r}+\widehat{c}_{u}\right)}{\widehat{m}_{r}-\widehat{m}_{b}} & , u_{d}\left(t_{k-1}\right)<u_{d}\left(t_{k}\right)\end{cases} \\
& u_{d \ell}\left(t_{k}\right)= \begin{cases}\frac{\widehat{m}_{\ell}\left(\widehat{m}_{t} \widehat{c}_{\ell}+\widehat{c}_{d}\right)}{\widehat{m}_{\ell}-\widehat{m}_{t}} & , u_{d}\left(t_{k-1}\right)>u_{d}\left(t_{k}\right) \\
\frac{\widehat{m}_{\ell}\left(\widehat{m}_{b} \widehat{c}_{\ell}+\widehat{c}_{u}\right)}{\widehat{m}_{\ell}-\widehat{m}_{b}} & , u_{d}\left(t_{k-1}\right)<u_{d}\left(t_{k}\right)\end{cases}
\end{aligned}
$$

as the coordinate points where an inner loop begins. For a given input $u_{d}$, the inverse output $v$ is then defined by the relation

$$
v\left(t_{k}\right)= \begin{cases}v\left(t_{k-1}\right) & \text { if } u_{d}\left(t_{k}\right)=u_{d}\left(t_{k-1}\right) \\ \frac{1}{m_{t}}\left(u_{d}\left(t_{k}\right)-\widehat{c}_{t}\right) & \text { if } u_{d}\left(t_{k}\right) \geq u_{3} \\ \frac{1}{m_{b}}\left(u_{d}\left(t_{k}\right)-\widehat{c}_{b}\right) & \text { if } u_{d}\left(t_{k}\right) \leq u_{4} \\ \frac{1}{m_{r}} u_{d}\left(t_{k}\right)+\widehat{c}_{r} & \text { if } u_{3} \geq u_{d}\left(t_{k}\right) \geq u_{d r} \\ \frac{1}{\widehat{m}_{\ell}} u_{d}\left(t_{k}\right)+\widehat{c}_{\ell} & \text { if } u_{4} \leq u_{d}\left(t_{k}\right) \leq u_{d \ell} \\ \frac{1}{m_{t}}\left(u_{d}\left(t_{k}\right)-\widehat{c}_{d}\right) & \text { if } u_{d \ell}<u_{d}\left(t_{k}\right)<u_{d r} \\ & \text { and } u_{d}\left(t_{k-1}\right)>u_{d}\left(t_{k}\right) \\ \frac{1}{m_{b}}\left(u_{d}\left(t_{k}\right)-\widehat{c}_{u}\right) & \text { if } u_{d \ell}<u_{d}\left(t_{k}\right)<u_{d r} \\ & \text { and } u_{d}\left(t_{k-1}\right)<u_{d}\left(t_{k}\right) .\end{cases}
$$




\section{Adaptive Hysteresis Inverse}

To facilitate the development of a suitable control law and adaptation scheme for updating the parameters in the model inverse, it is advantageous to consider a parameterization of the control error $u(t)-u_{d}(t)$ which isolates a parameterizable component from unknown disturbances. For the remainder of this development, we consider the case of symmetric hysteresis loops since this simplifies the discussion and is reasonably accurate for the milling application under consideration. Details regarding the control design can be found in $[9,10]$.

To specify the location of signals within both the hysteresis model and its inverse, we define indicator functions

$$
\begin{aligned}
& \chi_{t}(t)=\left\{\begin{array}{l}
1, \text { if } u(t)=m_{t} v(t)+c_{t} \\
0, \text { otherwise }
\end{array}\right. \\
& \widehat{\chi}_{t}(t)= \begin{cases}1, \text { if } v(t)=\frac{1}{m_{t}}\left(u_{d}(t)-\widehat{c}_{t}\right) \\
0, \text { otherwise }\end{cases}
\end{aligned}
$$

with similar definitions for the remaining components. For the symmetric case, the parameters and parameter estimates are specified as

$$
\begin{aligned}
& \theta_{h}^{*}=\left[m_{t}, c_{t}, m_{r}, m_{r} c_{r}\right]^{T} \\
& \theta_{h}(t)=\left[\widehat{m}_{t}(t), \widehat{c}_{t}(t), \widehat{m}_{r}(t), \widehat{m_{r} c_{r}}(t)\right]^{T}
\end{aligned}
$$

where ${\widehat{m_{r} c_{r}}}_{r}(t)=\widehat{m}_{r}(t) \widehat{c}_{r}(t)$. The error between parameters and estimates is then $\phi_{h}(t)=\theta_{h}(t)-\theta_{h}^{*}$. Finally, we define the regressor vector

$$
\begin{gathered}
\omega_{h}(t)=-\left[\left(\widehat{\chi}_{t}(t)+\widehat{\chi}_{c}(t)+\widehat{\chi}_{b}(t)\right) v(t),\left(\widehat{\chi}_{t}(t)-\widehat{\chi}_{b}(t)\right),\right. \\
\left.\left.\left(\widehat{\chi}_{r}(t)+\widehat{\chi}_{\ell}(t)\right) v(t),-\widehat{\chi}_{r}(t)+\widehat{\chi}_{\ell}(t)\right)\right]^{T}
\end{gathered}
$$

The control error $u(t)$ can then be expressed as

$$
u(t)=u_{d}(t)+\phi_{h}^{T}(t) \omega_{h}(t)+d_{h}(t) .
$$

The term $\phi_{h}^{T}(t) \omega_{h}(t)$ represents the parameterizable component of the error and, as established in [10], the disturbance $d_{h}(t)$ is bounded for all $t \geq 0$ if the parameter estimates are bounded and it reduces to zero when the parameter error is zero.

To generate the desired inputs $u_{d}(t)$, we employ the linear control law

$$
u_{d}(t)=\theta_{1}^{*} \omega_{1}(t)+\theta_{2}^{*} \omega_{2}(t)+\theta_{20}^{*} y(t)+\theta_{3}^{*} r(t)
$$

where

$$
\omega_{1}(t)=\frac{a(s)}{\Lambda(s)}\left[u_{d}\right](t) \text { and } \omega_{2}(t)=\frac{a(s)}{\Lambda(s)}[y](t) .
$$

Here $a(s)=\left(1, s, s^{2}, \ldots, s^{n-2}\right)^{T}$ and $\Lambda(s)$ is a monic Hurwitz polynomial of degree $n-1$ where $n$ is the degree of $P(s)$. Rather than obtaining the gains through the solution of the traditional Diophantine equation, we employed simplex-based optimization techniques.

To define the parameter update law, we note that the tracking error is $e(t)=y(t)-y_{m}(t)$ where $y_{m}(t)=$ $W_{m}(s)[r](t)$ with $W_{m}(s)$ stable and having the same relative degree as $G(s)$. We next define the quantities

$$
\begin{aligned}
& F(s)=\theta_{3}^{*-1} W_{m}(s)\left(1-\theta_{1}^{* T} a(s) / \Lambda(s)\right) \\
& \zeta_{h}(t)=F(s)\left[\omega_{h}\right](t) \\
& \xi_{h}(t)=\theta_{h}^{T}(t) \zeta_{h}(t)-F(s)\left[\theta_{h}^{T} \omega_{h}\right](t) \\
& \epsilon_{h}(t)=e(t)+\xi_{h}(t) .
\end{aligned}
$$

From $[9,10]$, we choose the update law

$$
\dot{\theta}_{h}(t)=-\frac{\Gamma_{h} \zeta_{h}(t) \epsilon_{h}(t)}{1+\zeta_{h}^{T}(t) \zeta_{h}(t)+\xi_{h}^{2}(t)}-\Gamma_{h} \Sigma\left(\theta_{h}\right) \theta_{h}(t)
$$

where $\Gamma_{h}$ is a symmetric, positive matrix and

$$
\Sigma\left(\theta_{h}\right)= \begin{cases}0 & \text { if }\left\|\theta_{h}(t)\right\|<M_{h} \\ \sigma_{0}\left(\frac{\left\|\theta_{h}(t)\right\|}{M_{h}}-1\right) & \text { if } M_{h} \leq\left\|\theta_{h}(t)\right\| \\ & \text { and }\left\|\theta_{h}(t)\right\|<2 M_{h} \\ \sigma_{0} & \text { if }\left\|\theta_{h}(t)\right\| \geq 2 M_{h}\end{cases}
$$

incorporates a priori knowledge of the upper bound $M_{h}$ on the Euclidean norm $\left\|\theta_{h}^{*}\right\|$ of $\theta_{h}^{*}$ and a design parameter $\sigma_{0}>0$.

\section{Numerical Example}

The performance of the adaptive control method is illustrated here in the context of a magnetostrictive transducer employed for high speed milling of out-of-round objects. As detailed in [8], the required tolerance for the cutting tip is \pm 1 micron.

The dynamics of the transducer are modeled by the transfer function $G(s)$ defined in (8) which has relative degree 1 . The physical parameters for the model and control design are compiled in Table 1 and are consistent with values reported in [3] for an actual transducer. The reference model was taken to be $W_{m}(s)=\frac{1}{s+2.2112 \times 10^{8}}$ which incorporates the prevalent transducer dynamics while facilitating implementation. For the control design, we employed the monic polynomial $\Lambda(s)=(s+3)^{3}$ along with $a(s)=\left(1, s, s^{2}\right)^{T}$.

The reference signal and transducer response obtained with the initial parameter values $\widehat{m_{t}}=6, \widehat{c_{t}}=5 \times 10^{5}$, $\widehat{m_{r}}=37, \widehat{c_{r}}=3 \times 10^{3}$ are plotted in Figure 4 and the tracking error is plotted in Figure 5. It is observed that the \pm 1 micron tolerances are achieved within six oscillations. For the cutting device, this is sufficient since cutting does not start immediately. Furthermore, the 
Table 1. Model parameters.

\begin{tabular}{|c|c|}
\hline$\rho$ & $9250 \mathrm{~kg} / \mathrm{m}^{2}$ \\
\hline$E$ & $3 \times 10^{10} \mathrm{~N} / \mathrm{m}^{2}$ \\
\hline$c_{D}$ & $3 \times 10^{6} \mathrm{Ns} / \mathrm{m}^{2}$ \\
\hline$M_{L}$ & $0.5 \mathrm{~kg}$ \\
\hline$k_{L}$ & $2 \times 10^{6} \mathrm{~N} / \mathrm{m}$ \\
\hline$c_{L}$ & $1 \times 10^{3} \mathrm{Ns} / \mathrm{m}$ \\
\hline$m_{t}=m_{b}$ & 8 \\
\hline$c_{t}=-c_{b}$ & $5.9 \times 10^{9}$ \\
\hline$m_{r}=m_{\ell}$ & 41.8 \\
\hline$c_{r}=-c_{\ell}$ & $3.14 \times 10^{3}$ \\
\hline$\Gamma_{h}$ & $2 \times 10^{11} I$ \\
\hline$\sigma_{0}$ & .15 \\
\hline$M_{h}$ & $7 \times 10^{5}$ \\
\hline
\end{tabular}

transducer response is devoid of the phase delays often associated with hysteretic operation. Finally, the spikes observed in the error are due to limitations in the piecewise linear Preisach model near saturation. If additional accuracy is required, these errors can be mitigated through the use of higher-order Preisach models or physical models such as the domain wall model $[2,3]$.

\section{Acknowledgements}

This research was supported in part by the Air Force Office of Scientific Research under the grant AFOSRF49620-01-1-0107.

\section{References}

[1] A. Adly, I. Mayergoyz and A. Bergqvist, "Preisach modeling of magnetostrictive hysteresis," Journal of Applied Physics, 69(8), pp. 5777-5779, 1991.

[2] F.T. Calkins, R.C. Smith and A.B. Flatau, "An energy-based hysteresis model for magnetostrictive transducers," IEEE Transactions on Magnetics, 36 (2), pp. 429-439, 2000.

[3] M.J. Dapino, R.C. Smith and A.B. Flatau, "A structural-magnetic strain model for magnetostrictive transducers," IEEE Transactions on Magnetics, 36(3), pp. 545-556, 2000.

[4] J. Nealis and R.C. Smith, "Partial Inverse Compensation Techniques for Linear Control Design in Magnetostrictive Transducers," Proc. SPIE, Smart Structures and Materials, 2001, to appear.

[5] P.M. Prenter, Splines and Variational Methods, Wiley, New York, 1975.

[6] J.B. Restorff, H.T. Savage, A.E. Clark and M. WunFogle, "Preisach modeling of hysteresis in Terfenol," J. Appl. Phys., 67(9), pp. 5016-5018, 1996.

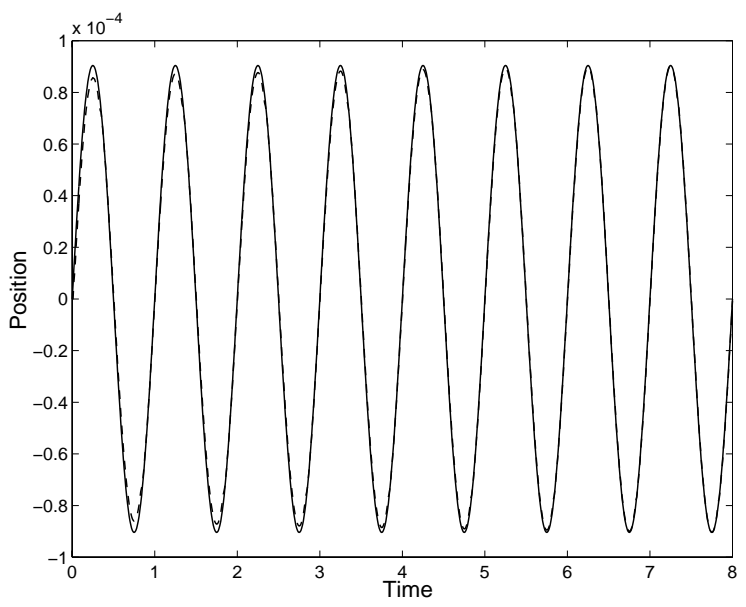

Figure 4. Reference $(-)$ and model response (- - ).

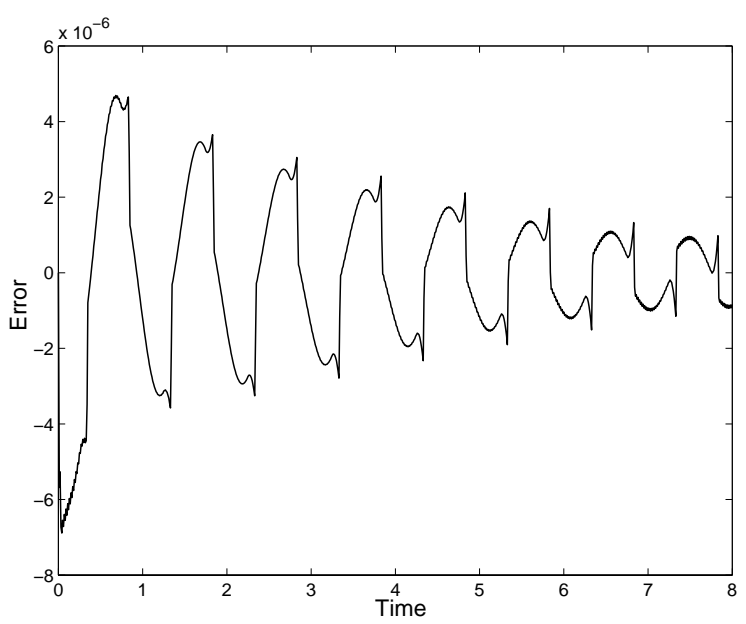

Figure 5. Tracking error for the transducer.

[7] R.C. Smith, "Hysteresis modeling in magnetostrictive materials via Preisach operators." J. of Mathematical Systems, Estimation and Control, 8(2), summary pp. 249-252, 1998.

[8] R.C. Smith, C. Bouton and R. Zrostlik, "Partial and full inverse compensation for hysteresis in smart material systems," Proceedings of the 2000 American Control Conference.

[9] G. Tao and P.V. Kokotović, "Adaptive control of plants with unknown hysteresis," IEEE Trans. on Automatic Control, 40(2), pp. 200-212, 1995.

[10] G. Tao and P.V. Kokotović, Adaptive Control of Systems with Actuator and Sensor Nonlinearities, John Wiley and Sons, New York, 1996.

[11] R. Venkataraman and P. Krishnaprasad, "A model for a thin magnetostrictive actuator," in Proceedings of the Conference on Information Sciences and Systems, 1998. 